\section{SAT0056 RHEUMATOID ARTHRITIS MAGNETIC RESONANCE IMAGING SCORE (RAMRIS) CAN PREDICT DAS-28 THERAPY RESPONSE AFTER 6 MONTHS: RESULTS OF THE GERMAN ARTHROMARK COHORT}

P. Sewerin ${ }^{1}$, S. Vordenbäumen ${ }^{1}$, C. Schleich ${ }^{2}$, R. Sengewein ${ }^{1}$, R. Brinks ${ }^{3}$, G. Pongratz ${ }^{3}$, J. Lesch ${ }^{1}$, L. Le ${ }^{4}$, U. Mansmann ${ }^{4}$, M. Schneider ${ }^{1}$, B. Ostendorf ${ }^{1}$ on behalf of ArthroMark Studygroup. ${ }^{1}$ Department for Rheumatology;

${ }^{2}$ Department for diagnostic and interventional Radiology;

${ }^{3}$ Hiller-Research-Center for Rheumatology, University Hospital Duesseldorf,

Düsseldorf; ${ }^{4}$ Institute for Medical Information Sciences, Biometry and

Epidemiology, Ludwig Maximilian University of Munich, Munich, Germany

Background: Remission is the ultimate goal in rheumatoid arthritis (RA). The absence of rheumatoid factors (RF) and /or anti-citrullinated protein (CCP) antibodies, no bone erosions on conventional x-rays, the presence of low disease activity, and early therapeutic intervention are established good prognostic markers. Magnetic resonance imaging (MRI) is a well evaluated imaging technic and is increasingly used in daily practice. In this study, we prospectively investigated the prognostic performance of high-field MRI and serological biomarkers 6 months after initiation of methotrexate in patients with early RA (eRA)

Objectives: To evaluate the value of high-resolution MRI of the hand as a prognostic marker for EULAR-response and remission after 6 month of MTX therapy in early RA patients

Methods: Prospective cohort study on the ArthroMark cohort using 3T MRI of the hand at baseline (V0) before initiating an MTX-therapy in eRA patients, after 3 months (V3) and after six months (V6). 28 patients ( $\varnothing 56.8$ years) with RF and/or CCP positive RA and a disease duration $<6$ months (mean16.3 weeks) fulfilling the 2010 ACR/EULAR criteria were examined. EULAR core set of variables were recorded: patient's global assessment of overall disease activity; number of tender and swollen joints, ESR and CRP. The following biomarkers were assessed by ELISA: Dkk-1, Osteoprotegerin, IL-22, MMP-3, TNF-Alpha and Neuropeptide-Y. Remission was defined as DAS28 $<2.6$ according to the ACR/EULAR remission criteria. MRI-scans were analysed by using OMERACT RA-MRI scoring system (RAMRIS). To adjust for intrapersonal correlation, we calculated generalized linear mixed models with time being recognized as a confounder in pretests.

Results: A low RAMRIS subscore for erosions $(p=0.019)$ or total RAMRIS score predicted response at V3 $(p=0.03)$. No significant results were found for the other imaging markers assessed for response prediction at either V 3 or V6. Concerning remission, low levels of RANKL at baseline were significantly associated with EULAR remission at V6 $(p=0.033)$. The other markers assessed did not show significant results at either V3 or V6.

In multivariate analyses, response was predicted more accurately with the inclusion of either RAMRIS ( $p$ value LR-test $=0.035$ ), RAMRIS synovitis subscore at MCP-2 ( $p$-value LR-test $=0.035$ ) or a combination of the two ( $p$-value LRtest $=0.042$ ). Remission was more accurately predicted when RANKL was considered with low RANKL improving the chance of remission. In contrast to response-prediction, MRI did not significantly add to the prediction model for remission.

Conclusions: Low RAMRIS scores or RAMRIS synovitis subscores at MCP-2 were predictive for therapy response after 6 months in our generalized mixed model. Baseline RANKL was able to significantly predict remission. Our data suggests that MRI and/or biomarkers may aid response prediction and facilitate patient selection for intensified therapy in the future.

Acknowledgements: ArthroMark study is funded by the Federal Ministry of Education and Researche (Bundesministerium für Bildung und Forschung, BMBF, FKZ 01EC1009A).

Disclosure of Interest: None declared

DOI: 10.1136/annrheumdis-2017-eular.3808

\section{SAT0057 PROSPECTIVE OBSERVATIONAL REAL-LIFE STUDY (STRATEGE) SHOWS THE EFFICACY OF TREAT-TO-TARGET STRATEGY AND METHOTREXATE MONOTHERAPY OPTIMIZATION IN PATIENTS WITH ESTABLISHED RHEUMATOID ARTHRITIS}

R.-M. Flipo $^{1}$, C. Gaujoux-Viala ${ }^{2,3}$, C. Hudry ${ }^{4,5}$, E. Zinovieva ${ }^{6}$ H. Herman-Demars ${ }^{6}$ on behalf of STRATEGE study observers. ${ }^{1}$ Rheumatology Department, Lille University Hospital Roger Salengro, Lille; ${ }^{2}$ Rheumatology Department, Nîmes Hospital, Nîmes; ${ }^{3}$ Montpellier University, Montpellier; ${ }^{4}$ Rheumatology Institute, ${ }^{5}$ Rheumatology Department, Cochin Hospital; ${ }^{6}$ Medical Department, Nordic Pharma, Paris, France

Background: Current guidelines consider MTX as initial gold standard treatment for patients (pts) with RA. They also propose various strategies for MTX inadequate responders, among which the most frequent are optimization of MTX therapy (alone or in combination with csDMARDs or bDMARDs).

Objectives: The objective of the trial was to explore the strategies applied in daily practice in RA pts with inadequate response to MTX.

Methods: STRATEGE was a prospective, observational, multicenter study. Main inclusion criteria were: confirmed RA (ACR 1987 or ACR/EULAR 2010 criteria) and treatment by MTX monotherapy with clinical, structural, functional and/or therapeutic evolution leading to therapeutic management modification. Data were obtained at 2 time-points: baseline and 6-month follow-up.
Results: Between Sept 2014 and July 2015, 176 rheumatologists, at 90\% with private practice, included 854 pts, 801 of which composed the analyzable baseline set. Pts baseline characteristics were [mean (SD)]: age: 57.4 (13.7) yrs; RA duration: 5.3 (6.7) yrs; DAS28: 4.0 (1.1), with the following distribution: <2.6 for $10 \%,>3.2$ for $74 \%$ and $>5.1$ for $16 \%$; HAQ: $1.1(0.84)$; and extra-articular features and erosive disease for respectively $10.5 \%$ and $39.9 \%$ of pts. All pts were receiving MTX monotherapy, orally for $67.6 \%$ and at mean (SD) dose of $14.2(4.1) \mathrm{mg} / \mathrm{wk}$ for oral and 16.6 (3.8) $\mathrm{mg} / \mathrm{wk}$ for parenteral administration. Concomitant treatment included corticosteroids for $45.8 \%$ of pts, at a mean (SD) dose of $8.2(6.3) \mathrm{mg} / \mathrm{d}$, and folic acid for $90.0 \%$. After the inclusion visit, MTX prescription has been identically maintained (dose and route) for $28.1 \%$ of pts, interrupted for $1.9 \%$ and modified for $70.0 \%$. Changes included dose increasing for $50.2 \%$, dose tapering for $1.8 \%$ and a route modification for $21.4 \%(88.2 \%$ oral -> parenteral). After inclusion visit, MTX oral versus (vs) parenteral balance was respectively $49.8 \%$ at mean (SD) dose $16.2(4.0) \mathrm{mg} / \mathrm{wk}$ vs $45.8 \%, 18.0$ (3.9) $\mathrm{mg} / \mathrm{wk}$. Biologic treatment was initiated for $14.6 \%$, in association with MTX for $95.7 \%$. Other csDMARD treatment was initiated for $1.2 \%$ in monotherapy and for $3.6 \%$ in association with MTX. The reasons for treatment modification were mainly active RA $(72.0 \%)$, worsening of clinical and biologic parameters $(31.4 \%)$, radiographic progression $(14.5 \%)$, remission not achieved $(12.4 \%)$, steroid dependence $(11.3 \%)$, and MTX intolerance $5.0 \%$. Six-month follow-up results show that all the active treatment strategies were significantly and equally successful in improving disease activity (Table).

Table 1

\begin{tabular}{lccccc}
\hline & $\mathrm{N}$ & DAS28 Baseline & DAS28 (M6) & D DAS28 & $p$ \\
\hline MTX unchanged (Ref.) & 126 & $3.4(1.3)$ & $2.5(1.0)^{\star}$ & $-0.8(1.2)$ & - \\
MTX optimization & 519 & $4.0(1.0)$ & $2.9(1.2)^{\star}$ & $-1.1(1.3)$ & 0.10 \\
bDMARDs & 117 & $4.6(1.1)$ & $3.2(1.1)^{\star}$ & $-1.4(1.3)$ & 0.22 \\
csDMARDs & 39 & $4.3(1.2)$ & $3.1(1.3)^{\star}$ & $-1.3(1.4)$ & 0.37 \\
\hline
\end{tabular}

Data presented: mean (SD). ${ }^{*} p<0.0001$ (M6 vs baseline), ANCOVA, adjusted for DAS28 at baseline.

Conclusions: Consistently with all current guidelines, results of the large prospective observational study STRATEGE reveal an important place held by initial MTX treatment optimization before initiation of a biotherapy and emphasize the importance of treat-to-target strategy.

Disclosure of Interest: R.-M. Flipo Consultant for: Nordic Pharma, C. GaujouxViala Consultant for: Nordic Pharma, C. Hudry Consultant for: Nordic Pharma, E. Zinovieva Employee of: Nordic Pharma, H. Herman-Demars Employee of: Nordic Pharma

DOI: 10.1136/annrheumdis-2017-eular.2707

\section{SAT0058 EFFECTS OF BARICITINIB ON PATIENTS WHO STOP METHOTREXATE MONOTHERAPY AND SWITCH TO BARICITINIB MONOTHERAPY}

R. Fleischmann ${ }^{1}$, T. Takeuchi ${ }^{2}$, M. Schiff ${ }^{3}$, D. Schlichting ${ }^{4}$, L. Xie ${ }^{4}$, M. Issa ${ }^{4}$, I. Stoykov ${ }^{4}$, J.R. Lisse ${ }^{4}$, P. Martinez-Osuna ${ }^{4}$, T. Rooney ${ }^{4}$, C.A. Zerbini ${ }^{5} .{ }^{1} U$ Texas SW Medical Center, Dallas, United States; ${ }^{2}$ Keio University, Tokyo, Japan; ${ }^{3}$ Univ Colorado Hospital Rheum, Colorado; ${ }^{4}$ Eli Lilly and Company, Indianapolis, United States; ${ }^{5}$ Univ Sao Paolo, Sao Palo, Brazil

Background: Baricitinib (bari) is a reversible oral Janus kinase (JAK) inhibitor with selectivity for JAK1/JAK2 in development for treatment of patients (pts) with active rheumatoid arthritis (RA). In the 52-week (wk) Phase 3 RA-BEGIN study of MTX-naïve pts, there were 3 arms: bari $4 \mathrm{mg}$ once daily (QD), methotrexate (MTX) up to $20 \mathrm{mg}$ weekly $(\mathrm{QW})$, and the combination of bari plus MTX (bari+MTX). Nonresponders were rescued from week 24 onwards by receiving bari+MTX, regardless of original treatment. Bari monotherapy showed superior efficacy compared to MTX monotherapy and similar clinical efficacy to bari+MTX

Objectives: Efficacy and safety were evaluated in pts from RA-BEGIN who switched from MTX or bari+MTX therapy to bari monotherapy upon entering the long-term extension (LTE) study (RA-BEYOND).

Methods: In RA-BEGIN, 588 pts were randomised 4:3:4 to MTX, bari monotherapy $4 \mathrm{mg}$, or bari+MTX. At Wk 52, pts could enter the LTE; all pts received bari $4 \mathrm{mg}$ monotherapy. MTX could be added in the LTE by investigator decision. Seventy-seven percent of pts (451/588) enrolled in the LTE, of whom 423 had not been rescued in RA-BEGIN. This post hoc analysis evaluated clinical efficacy of pts who continued bari monotherapy compared to those in whom MTX was added within the first 24 wks of the LTE.

Results: Of these 423 pts, $200(47 \%)$ remained on monotherapy at Wk 24 of the LTE and 223 pts started on MTX before wk 24. Most (193) had initiated MTX within 4 wks of starting the LTE study, evenly balanced from the 3 original arms of RA-BEGIN. Across study arms, pts who had MTX added in the LTE had worse disease control upon entry and during the LTE. Through 24 wks, statistically significant improvement in disease state was observed in the MTX-to-bari group regardless of whether or not MTX was added back. In the bari-to-bari monotherapy group, the addition of MTX led to lowered disease activity, which was statistically significant. No statistically significant changes in disease activity were observed in the pts who were switched from bari+MTX to bari monotherapy regardless of additional MTX therapy (Table 1). Exposure-adjusted incidence rates for total treatment-emergent adverse events, including non-serious infections, were lowest in the MTX-to-bari group. Clinically significant or consistent differences in SIE, 
SAEs, or AEs leading to study drug discontinuation were not seen in any of the arms, whether MTX was added or not.

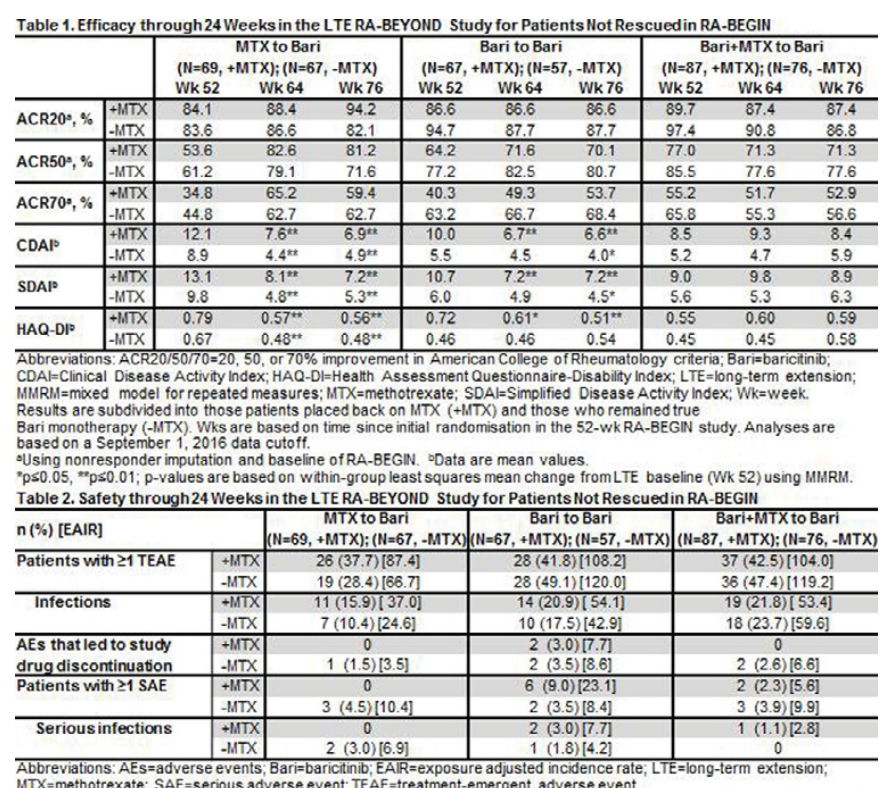

Analyses are based on a September 1,2016 data cutoff. Data are n (\%) [EAIR].

Conclusions: Switching from MTX to bari monotherapy, maintaining bari monotherapy was associated with improvements in depths of disease control during the initial 24 wks post-switch. Disease control did not significantly change after withdrawal of MTX from combination therapy. Pts who entered the LTE with suboptimal disease control after treatment with bari monotherapy may benefit from the addition of MTX. Discontinuation of MTX in pts treated with combination during the index study was associated with maintenance of response. There were no differences in important measures of safety including events that are serious or led to discontinuation.

Disclosure of Interest: R. Fleischmann Grant/research support from: AbbVie, Amgen, AstraZeneca, Bristol-Myers Squibb, Celgene, Genetech, GlaxoSmithKline, Janssen, Eli Lilly and Company, Merck, Pfizer, Regeneron, Roche, Sanofi Aventis, UCB, Consultant for: AbbVie, Akros, Amgen, Bristol-Myers Squibb, Celgene, Genentech, GSK, Janssen, Eli Lilly and Company, Pfizer, Sanofi-Aventis, UCB, T. Takeuchi Consultant for: Pfizer Japan, Astra Zeneca KK, Eli Lilly Japan KK, Novartis Pharma KK, Daiichi Sankyo Co Ltd, Nipponkayaku Ltd, Janssen Pharma KK, Merck Serono Ltd, Takeda Pharma Ltd, Mitsubishi Tanabe Pharma, Astellas Pharm, Abbvie GK, Bristol-Myers KK, Asahi Kasei Medical KK, Speakers bureau: Celtrion, Nipponkayaku Ltd, Pfizer Japan, UCB Japan, Daiichi Sankyo Ltd, Takeda Pharma Ltd, Chugai Pharma Ltd, Abbvie GK, Bristol-Myers KK, Eisai Ltd, Mitsubishi Tanabe Pharma, Janssen Pharma KK, Astellas Pharma, M. Schiff Consultant for: Abbvie, Amgen, Antares, BMS, Eli Lilly and Company, J\&J, Novartis, Novo Niordisk, Pfizer, Roche, UCB, Speakers bureau: Abbvie, BMS, D. Schlichting Employee of: Eli Lilly and Company, L. Xie Employee of: Eli Lilly and Company, M. Issa Employee of: Eli Lilly and Company, I. Stoykov Employee of: Eli Lilly and Company, J. Lisse Employee of: Eli Lilly and Company, P. Martinez-Osuna Employee of: Eli Lilly and Company, T. Rooney Employee of: Eli Lilly and Company, C. Zerbini Grant/research support from: Pfizer, Novartis, Eli Lilly and Company, Merck, Sanofi, Amgen, Celtrion, Consultant for: Merck, Pfizer, Sanofi, Eli Lilly and Company

DOI: 10.1136/annrheumdis-2017-eular.1334

\section{SAT0059 HIGH RADIOGRAPHIC PROGRESSION IS RARE AND GETS LESS FREQUENT IN RA PATIENTS OF THE SWISS SCQM COHORT FROM 1998 TO 2015}

$\underline{\text { R. Mueller }}^{1}$, R. Thalmann ${ }^{1}$, H. Schulze-Koops ${ }^{2}$, N. Graf ${ }^{3}$, J. von Kempis ${ }^{1}$. ${ }^{1}$ Division of Rheumatology, Kantonsspital St. Gallen, St. Gallen, Switzerland;

${ }^{2}$ Rheumaeinheit, Klinikum der Universität München, Campus Innenstadt, Munich, Germany; ${ }^{3}$ Graf Biostatistics, Winterthur, Switzerland

Background: The primary goal of current therapeutic strategies in RA is to control disease activity and at the same time to prevent radiographic progression. A disconnect between a missing radiographic progression and higher disease activity under treatment with biologic agents has been discussed. In general, analyses of radiographic progression in RA rather focus on radiographic nonprogression or repair and high radiographic progression in spite of therapy has, to our knowledge not been analysed in detail in the last years, neither in RCTs nor in cohort studies.

Objectives: To analyse the occurrence of high radiographic destruction and it's development in the era of biologic treatment.
Methods: We included all RA patients from the Swiss registry SCQM with at least two subsequently scored radiographs. Radiographic destruction was scored with the Ratingen erosion score and calculated by dividing the change of Ratingen scores by time ( $>6$ months) between two subsequent radiographs. To analyse high radiographic progression we looked for the height and time of the highest (peak) radiographic progression in every individual patient for the analysis. The individual peak radiographic progression was analysed in groups as change of Ratingen scores/year: $0-\leq 10,10-\leq 20,20-\leq 30,>30$ (groups $1-4$ ). The time point of the individual peak radiographic progression was analysed per group (follow up 1998 - 2015). The baseline disease characteristics were compared using standard descriptive statistics (Kruskal-Wallis or Chi-square tests).

Results: 4'033 patients were included into the analysis. 3'049 patients had a peak radiographic progression between 0 and $\leq 10$ /year, 773 between 10 and $\leq 20,150$ between 20 and $\leq 30$, and 61 of $>30$. All patient groups were within the same age range (mean: $56.5-60.5$ years). Rheumatoid factor and ACPA were more frequent in patient groups with higher peak radiographic progression (RF: 73.6, 80.0, 88.9, 90.0; ACPA: 66.8, 73.4, 74.3, 82.1, groups $1-4$, respectively). When the rate of radiographic progression before and after peak progression was analysed, $69.7 \%, 74.7 \%, 76.9 \%$, and $93.3 \%$ of the patients had a radiographic progression of $25 \%$ or lower as compared to peak progression before and $76.1 \%$, $81.8 \%, 91.1 \%$, and $93.8 \%$ after this peak progression, respectively for patients in groups 1 to 4 (Figure A).

The disease activity, as assessed by DAS 28 , was significantly higher in all patient groups before peak progression and lower thereafter (Figure $B, p<0.001$ ). Average HAQ-DI scores increased after peak radiographic progression in group 4 (Figure $C, p=0.005$ ) whereas it is stable or even decreases among the patients of the other patient groups.

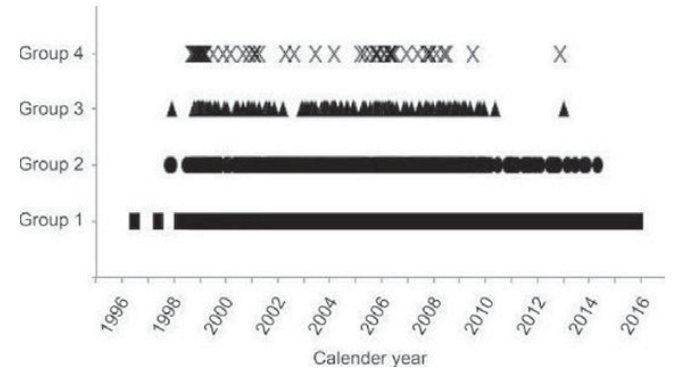

Conclusions: High peak radiographic progression is a rear phenomenon that gets less frequent in patients over the last years of observation. This may be an effect of modern therapy. Among the patients with high radiographic progression, (1) a greater delay between diagnosis and first symptoms was found and (2) the use of biologic agents was less frequent. These data suggest that the decreasing frequency of very high radiographic peak progression in the Swiss RA patients reflect a more effective therapeutic approach in the last years.

Disclosure of Interest: None declared

DOI: 10.1136/annrheumdis-2017-eular.4232

\section{SAT0060 RESPONSE TO CONVENTIONAL SYNTHETIC DMARDS DIFFERS DEPENDING ON RHEUMATOID FACTOR LEVELS IN ANTI-CITRULLINATED POSITIVE PATIENTS WITH EARLY RHEUMATOID ARTHRITIS}

S. Bugatti, A. Manzo, G. Zanframundo, F. Benaglio, G. Sakellariou, C. Montecucco, R. Caporali. Rheumatology and Translational Immunology Research Laboratories (LaRIT) and Early Arthritis Clinic, Division of Rheumatology, University of Pavia, IRCCS Policlinico San Matteo Foundation, Pavia, Italy

Background: In rheumatoid arthritis (RA), several autoantibody characteristics, including specifities, levels and isotypes, may influence their pathogenic properties and impact on clinical presentation and outcomes of the disease $(1,2)$. In particular, classical IgM rheumatoid factor (RF) may boost inflammation triggered by anti-citrullinated protein antibodies (ACPA) (3)

Objectives: To investigate whether RF impacts on disease characteristics and response to therapy in ACPA-positive early RA patients treated with conventional synthetic disease modifying drugs (csDMARDs)

Methods: 574 early RA patients consecutively enrolled in our Early Arthritis Clinic between 2005 and 2014 were included. Patients had symptoms' duration $<12$ months, were glucocorticoid- and DMARD-naïve, and fulfilled RA criteria at inclusion. IgM RF and IgG ACPA were determined in baseline sera by immunonephelometry and a second-generation EliA CCP assay respectively. Autoantibody levels were considered high when $>3$ times the upper limit of normal (ULN). Patients were treated with incremental doses of methotrexate according to a treat-to-target strategy aiming at low disease activity (LDA, DAS28 $\leq 3.2$ ). The associations between autoantibody specifities and levels and the achievement of LDA and disease remission (DAS28<2.6) over 6 months were investigated by Cox regression.

Results: 360 patients tested RF and/or ACPA positive (31.9\% single RF-positive, $10.3 \%$ single ACPA-positive, $57.8 \%$ ACPA and RF double-positive) and were 